\title{
Anabases
}

ANABASES Traditions et réceptions de l'Antiquité

$20 \mid 2014$

Varia

\section{Homère et Giovanni Pascoli}

\section{Cristina Noacco}

\section{OpenEdition}

Journals

Édition électronique

URL : http://journals.openedition.org/anabases/5024

DOI : 10.4000/anabases.5024

ISSN : 2256-9421

\section{Éditeur}

E.R.A.S.M.E.

\section{Édition imprimée}

Date de publication : 1 novembre 2014

Pagination : 193-210

ISSN : 1774-4296

\section{Référence électronique}

Cristina Noacco, « Homère et Giovanni Pascoli », Anabases [En ligne], 20 | 2014, mis en ligne le 01

novembre 2017, consulté le 20 octobre 2019. URL : http://journals.openedition.org/anabases/5024 DOI : 10.4000/anabases.5024 


\section{Homère et Giovanni Pascoli ${ }^{1}$}

Cristina Noacco

[...] Noi, s'è quello che s'è : una tempra d'eroici cuori, sempre la stessa : affranti dal tempo e dal fato, ma duri sempre in lottare e cercare e trovare né cedere mai $^{2}$.

EN 2012, AU MOMENT OƯ SE DÉROULAIT CETTE MANIFESTATION consacrée aux traductions des poèmes homériques en hexamètres, on célébrait le centenaire de la mort de Giovanni Pascoli (1855-1912), lecteur et traducteur d'Homère d'exception. Au tournant du $\mathrm{Xx}^{\mathrm{e}}$ siècle, ce poète a élaboré une théorie pour la composition de vers métriques en italien ${ }^{3}$, accompagnée d'un choix d'extraits de poèmes grecs et latins.

1 J'exprime toute ma reconnaissance à $M$. Francesco Zambon, qui est à l'origine de ce travail, à MM. Marco Bianchi et Daniele Ventre, qui m’ont guidée et conseillée dans le domaine de la métrique quantitative en italien et à MM. Paul François et André Bouvet qui ont relu et corrigé cette contribution.

2 G. PAscoli, Ulisse, in Sul limitare. Prose e poesie scelte per la scuola italiana, MilanoPalermo-Napoli, Remo Sandron Editore, s. d., $4^{\mathrm{e}}$ éd. (1 ${ }^{\mathrm{re}}$ éd. : 1900), p. 400. Traduction des derniers vers du poème Ulysses, d'Alfred Tennyson, Poems, 1842 : [...] That which we are, we are; / One equal temper of heroic hearts, / Made weak by time and fate, but strong in will / To strive, to seek, to find, and not to yield.

3 Cf. M. Valgimigli, "Nascita dell'esametro pascoliano ", in ID., Uomini e scrittori del mio tempo, Florence, Sansoni, 1965, p. 211-214 ; G. ConTINI, «Innovazioni metriche italiane fra Otto e Novecento ", in ID., Varianti e altra linguistica. Una raccolta di saggi (19381968), Turin, Einaudi, 1970, p. 587-599 ; F. AudisIO, "Metrica "neoclassica" e metrica italiana ", La rassegna della letteratura italiana 3 (settembre-dicembre 1995), p. 34-91 ; E. PASQUinI, "Pascoli vs Carducci : due modalità di traduzione ", in Teorie e forme del tradurre in versi nell'Ottocento fino a Carducci, Atti del convegno internazionale, Lecce, 2-4 ottobre 2008, a cura di A. Carrozzini, Galatina, Congedo Editore, 2010, p. 363-377. 
Ma contribution vise tout d'abord à exposer les aspects essentiels et les limites de la théorie pascolienne. Quelques exemples tirés de son anthologie scolaire Sul limitare, relatifs au premier chant de l'Iliade, me permettront ensuite de souligner les choix stylistiques et linguistiques qui ont guidé son travail de traduction ${ }^{4}$. Des remarques sur l'évolution de l'hexamètre dans la poésie de cet auteur, ainsi que dans deux autres traductions en vers isométriques italiens de l'Iliade, élaborées l'une au début du Xx ${ }^{\mathrm{e}}$ siècle par Manlio Faggella (1923) et l'autre, récemment, par Daniele Ventre (2011), clôtureront ce bref parcours.

\section{L'hexamètre italien de Giovanni Pascoli : approches théoriques}

Avant Giovanni Pascoli, plusieurs auteurs avaient élaboré des traductions italiennes en vers ou en prose, partielles ou complètes, des poèmes homériques : Melchiorre Cesarotti, Ugo Foscolo, Vincenzo Monti, Ippolito Pindemonte sont les plus connus. Mais tandis qu'on ne trouve chez ces auteurs aucune tentative pour concilier la langue accentuelle italienne avec le principe quantitatif de l'hexamètre ancien, d'autres expériences de métrique quantitative en italien avaient été menées, à partir $\mathrm{du} \mathrm{XV}^{\mathrm{e}}$ siècle, notamment par Leonardo Dati, Leon Battista Alberti, Claudio Tolomei et Tommaso Campanella ${ }^{5}$. À la suite de ces tentatives décevantes, les poètes italiens avaient renoncé à imiter rigoureusement la structure quantitative du vers gréco-latin en italien, du fait que la quantité ne jouait plus, dans cette langue, de rôle distinctif, et ils avaient de préférence employé des vers de la tradition italienne. Cette solution consistait à lire le vers ancien d'après les accents grammaticaux des mots et à en garder le rythme seulement en fin de vers ${ }^{6}$. Une dernière solution d'adaptation était apparue en Allemagne :

4 Les traductions (notamment homériques) de Pascoli ont suscité de nombreux travaux critiques. Voir B. Croce, G. Pascoli, Bari, Laterza, 1956 (1920) ; G. Devoto, «Problemi delle traduzioni pascoliane ", Studi per il centenario della nascita di G. Pascoli pubblicati nel cinquantenario della morte. Convegno bolognese (28-30 marzo 1958), 3 vol., Bologne, Commissione per i testi della lingua, 1962, t. II, p. 57-67 ; P. Giannini, "Le traduzioni "metriche" di G. Pascoli ", in Teorie e forme del tradurre in versi..., p. 379-396 ; N. Gardini, «An Ancient Soul in a New Body : Giovanni Pascoli’s Homeric Translations ", Italian Studies 66/1 (2011), p. 59-75 ; M. BIANCHI, "Oltre il limitare : l'Omero "adulto" di Giovanni Pascoli. Le traduzioni pascoliane dell' Iliade e la questione della metrica neoclassica ", Rivista pascoliana 23 (2011), p. 9-30. Pour une bibliographie plus détaillée, voir BIANCHI, p. 10.

5 Voir M. Geymonat, "Osservazioni sui primi tentativi di metrica quantitativa italiana ", Giornale Storico della Letteratura Italiana 143 (1966), p. 378-389 et P. G. Beltrami, La metrica italiana, Bologne, Il Mulino, 1994 (1991), p. 126-136 ; 195-206.

6 C'est la solution qu'adoptent, à partir de l'exemple de Gabriello Chiabrera, Paolo Rolli et Giovanni Fantoni et qui représente le fondement théorique du "vers barbare " de Giosué 
elle consistait à lire les vers gréco-latins d'après leur scansion métrique, en faisant correspondre les ictus avec des syllabes toniques et les mesures brèves avec des syllabes atones, sans toutefois faire violence à la structure de la langue moderne ${ }^{7}$.

En s'inscrivant dans cette nouvelle tendance, Giovanni Pascoli a mis en place, dans des textes qui datent du début du $\mathrm{xx}^{\mathrm{e}}$ siècle (Nota per gl'insegnanti, Regole di metrica neoclassica et Lettera a Giuseppe Chiarini $^{8}$ ), un système d'adaptation de l'hexamètre homérique en italien fondé sur trois points essentiels : la critique du "vers barbare " de Giosué Carducci, le choix de l'hexamètre dactylique et l'importance de l'intonation pour restituer le rythme de l'hexamètre en italien.

\section{La critique du « vers barbare » de Giosué Carducci}

Dans la Lettera a Giuseppe Chiarini, Pascoli présente avant tout sa perception du rythme tel qu'il apparaît dans les poèmes d'inspiration ancienne de Giosué Carducci, les Odes barbares ${ }^{9}$ : cet auteur y élabore un rythme poétique qui évoque les modèles métriques de la poésie grecque (essentiellement l'hexamètre et le pentamètre) grâce à l'association de vers provenant de la tradition littéraire italienne, notamment les vers de sept et de neuf syllabes ${ }^{10}$. Voici, par exemple, le début du premier poème du recueil, L'Aurora, composé en distiques élégiaques. L'hexamètre « barbare » du premier vers est formé, comme prévu, d'un settenario et d'un novenario:

Tu sali e baci, o dea, // co'l rosëo fiato le nubi

baci de' marmorëi templi le fosche cime.

En effet, la règle qu'adopte Giosué Carducci dans son adaptation de l'hexamètre et du pentamètre consiste à maintenir en italien le même nombre de syllabes ${ }^{11}$ qu'en grec et en latin, mais parfois seulement les mêmes accents (notamment dans les positions finales de l'hexamètre).

Pour ce qui est de l'hexamètre dactylique, Carducci transpose, dans la plupart des cas, le deuxième hémistiche par un vers de neuf syllabes avec des accents sur les $2^{\mathrm{e}}$, $5^{\mathrm{e}}$ et $8^{\mathrm{e}}$ positions, ce qui donne au vers un rythme comparable à celui de l'hexamètre,

Carducci, appelé ainsi en raison de son éloignement des modèles anciens.

7 Cf. Audisio, p. 34 et le site Treccani.it, L'enciclopedia italiana, s. v. "Barbara, metrica ". URL : http://www.treccani.it/enciclopedia/metrica-barbara/ (consulté le 26 janvier 2013).

8 G. Pascoli. Poesie e prose scelte, Progetto editoriale, introduzioni e commento di C. Garboli, 2 vol., Milan, Mondadori, 2002, t. II, p. 136-139 et p. 177-290.

9 G. CARDUCCI, Odi barbare, introduction, notices et commentaires par A. Ricklin, Paris, Les Belles Lettres, 1950.

10 Il s'agit, pour utiliser la terminologie italienne, du settenario et du novenario.

11 C'est-à-dire de 13 à 17 syllabes pour l'hexamètre et de 12 à 14 syllabes pour le pentamètre, compte tenu des substitutions permises dans ces vers. 
même sans tenir compte de la quantité, tandis qu'il utilise un vers de sept syllabes (moins souvent, de cinq ou six) pour transposer le premier hémistiche ${ }^{12}$. Dès lors, il peut arriver, mais sans que l'effet produit soit constant ni parfait, que la construction de l'hexamètre barbare de Carducci reproduise le schéma rythmique ancien, en particulier lorsque la première syllabe du vers est accentuée, comme on le voit dans le vers suivant :

Quándo a le | nóstre | cáse // la | díva se | véra di | scénde ${ }^{13}$.

Voici en revanche un exemple de "vers barbare » où le rythme repose sur l'évocation musicale de l'hexamètre, et non sur la correspondance exacte des accents. Il est composé d'un vers de cinq syllabes et d'un décasyllabe :

\section{E molli d'auree // ginestre si paravano i colli.}

Ce sont des exemples de ce genre qui font dire à Giovanni Pascoli que dans les poèmes de Carducci il perçoit la musicalité des vers grecs par un rythme indirect (il oppose un ritmo proprio à un ritmo riflesso), de même que, dit-il, les cigales frémissent par des bruits qui ne sont pas réguliers mais qui, entendus dans leur ensemble, donnent l'impression d'un rythme où des syllabes longues alternent avec des brèves ${ }^{14}$. Pascoli veut faire autre chose : reproduire en italien les schémas métriques anciens, en respectant le nombre de syllabes et la position des accents, ainsi que, d'après sa théorie métrique, la quantité. Il justifie sa position en expliquant que, pour lui, la langue italienne peut être considérée comme une langue non seulement accentuelle mais aussi quantitative, et il offre des arguments précis pour justifier la distinction qu'on peut faire en italien entre syllabes longues et syllabes brèves ${ }^{15}$.

À la différence du choix de traduction des mètres anciens proposé par Carducci, Pascoli, dans sa présentation des traductions homériques (68 extraits tirés de l'Iliade et 58 extraits de l'Odyssée), adopte donc la lecture rythmique-quantitative de l'hexamètre qu'il avait déjà expérimentée, encore jeune, dans la vulgarisation des cent premiers vers de la Batrachomyomachie ${ }^{16}$.

12 Je résume ainsi les considérations de Pietro G. Beltrami au sujet de la métrique des Odi barbare de Carducci, p. 131-134 et 199-200. Cf. les analyses de Cesare Garboli dans G. Pascoli. Poesie scelte e prose, t. I, p. 362.

13 Ici, comme dans les vers traduits du grec qui sont cités plus loin, je marque par des accents aigus les syllabes qui correspondent aux mesures longues de l'hexamètre.

14 Regole di metrica neoclassica, in G. Pascoli. Poesie e prose scelte, t. II, p. 203.

15 Le système élaboré par Pascoli afin de reconnaître les syllabes longues et les syllabes brèves en italien est présenté dans ses Regole..., p. 177-290. L'analyse la plus approfondie de ce système métrique a été proposée par AuDISIO, p. 42-91.

16 G. PASCOLI, Volgarizzamento del principio della Batracomiomachia, in Poesie, con un avvertimento di A. Baldini, 2 vol., Milan, Mondadori, 1971 [1939], t. II, p. 1679, v.1-4. Il est intéressant de remarquer que, bien que fondé sur des critères de traduction différents 


\section{L'hexamètre dactylique de Giovanni Pascoli}

La vulgarisation de la Batrachomyomachie constitue un exercice scolaire que Giovanni Pascoli avait présenté à son maître, le même Giosué Carducci, pendant l'année 1880-1881.

C'éra una $\mid$ vólta...' un $\mid$ tópo, che, | pér una $\mid$ dónnola es $\mid$ séndo

Mórto di $\mid$ séte, a un pa $\mid$ dúle si $\mid$ trásse, e sfio $\mid$ rávane | l'ácqua

Cól deli $\mid$ cáto suo $\mid$ ménto gus $\mid$ tándo quel $\mid$ miéle ; e lo $\mid$ víde

Ún godin $\mid$ brágo so $\mid$ nánte, che $\mid$ quésto dis $\mid$ córso gli $\mid$ ténne [...].

Dans l'édition Zanichelli de Traduzioni e riduzioni datée de 1920, sur la reproduction photographique de l'original, on peut même voir de petites marques sur des lettres ou des syllabes : ces marques étaient destinées à guider la lecture du texte, et devaient permettre au lecteur de repérer les hiatus et les diphtongues, de manière à rendre leur rythme aux dactyles et aux spondées. Ce détail montre que, dès ce premier exercice de traduction, Pascoli avait rejeté les combinaisons des vers italiens adoptées par Carducci dans ses "vers barbares ", pour retrouver le rythme des pieds de la poésie antique ${ }^{17}$. Face à l'enseignement du maître, cette première expérience d'adaptation de l'hexamètre en italien constitue une alternative à la fois courageuse et polémique de la part du jeune poète, qui maintiendra le cap tout au long de ses futures expériences de traduction ${ }^{18}$.

Dans l'introduction à ce travail, Pascoli justifie son ambition en disant qu'il a " presque toujours évité les pieds spondaïques, du fait qu'on ne peut empêcher qu'ils soient soit trochaïques soit iambiques ${ }^{19}$ ". Par la suite, le poète confirme son choix de traduction dans un texte qu'il rédige à l'intention des professeurs de latin (Nota per gl'insegnanti) :

Notre langue a quelque réticence à accueillir la mesure des pieds. La raison principale en a été indiquée par Mommsen (cité par Chiarini dans son merveilleux essai sur la métrique des Odes barbares [de Giosué Carducci]) : elle n’a pas de spondées. Cela est vrai.

En fait, cela n'est pas tout à fait vrai, voire n'est pas vrai du tout, comme on le verra dans un petit livre de moi qui va sortir bientôt. Pour l'heure, admettons que cela soit vrai. Eh bien ? Faisons alors seulement des dactyles. C'est ce que je pensais il y a deux ans et c'est ce que j’ai fait : les hexamètres sont ici presque uniquement composés de dactyles ${ }^{20}$.

de ceux de son maitre, cet exercice scolaire avait reçu de Giosué Carducci l'appréciation "Très bien " (Giannini, p. 382).

17 Valgimigli, p. 212-213.

18 Cf. Audisio, p. 37.

19 Ibid., p. 39. En raison de contraintes éditoriales, je ne transcris pas ici les citations dans leur langue d'origine. Sauf mention contraire, les traductions de l'italien sont personnelles.

20 G. Pascoli. Poesie e prose scelte, t. II, p. 137. 
Giacomo Devoto a défini l'hexamètre pascolien " comme une succession rythmique composée généralement de dix-sept syllabes, avec les temps forts normaux placés sur les $1^{\mathrm{e}}, 4^{\mathrm{e}}, 7^{\mathrm{e}}, 10^{\mathrm{e}}, 13^{\mathrm{e}}, 16^{\mathrm{e}}$ syllabes, et comportant la possibilité de réduire les intervalles de trois à deux syllabes, grâce au prolongement d'un temps fort ${ }^{21}$ ". Cela revient à reconnaître que l'hexamètre pascolien est constitué de manière presque systématique de pieds dactyliques, ce qui représente la différence majeure entre l'hexamètre homérique et son adaptation en italien : en voulant composer des vers formés uniquement de dactyles, Pascoli s'éloigne de la structure de l'hexamètre homérique, où le spondée est assez fréquent ${ }^{22}$.

Toutefois, le spondée n'est pas totalement absent de ses traductions. Ainsi, en se servant de la possibilité d'allonger certaines syllabes qui portent un accent grammatical primaire (syllabes toniques) ou secondaire (syllabes protoniques et méta-toniques), Pascoli parvient à construire des pieds spondaïques à la manière des poètes allemands : on remarque alors la présence d'un spondée en $4^{\mathrm{e}}$ position, comme par exemple au v. 1 :

L’ira, o Déa, tu cánta // del Péleíade Achille.

ou, moins souvent, en deuxième position, comme au v. 6, où l'on trouve également un spondée en $4^{\mathrm{e}}$ position :

sino d'allór che prima // si séparárono in lótta.

En définitive, l'hexamètre pascolien repose sur une série de mots proparoxytons (portant l'accent sur la syllabe antépénultième); il imite la structure du dactyle et de mots (très rares) portant l'accent à la fois sur l'avant-dernière syllabe et sur la dernière, ce qui donne ainsi l'impression d'un spondée ${ }^{23}$.

Quant à la césure, obligatoire dans le vers dactylique, Pascoli rappelle qu'elle peut être placée après la troisième ou après la deuxième et la quatrième arsis (le temps fort ${ }^{24}$, ou bien prendre la forme de la trochä̈que troisième ${ }^{25}$, ce qui représente le cas le plus fréquent dans les poèmes homériques, ainsi que la solution la plus utilisée dans ses traductions :

Fúnebre, cáusa agli Achéi /l già d’infiniti dolóri.

Les fondements de la théorie de l'adaptation de l'hexamètre homérique à la langue italienne étaient jetés.

21 Devoto, p. 67.

22 Dans son analyse métrique des traductions homériques de Giovanni Pascoli, Marco Bianchi est parvenu à la même conclusion (p. 17).

23 Valgimigli, Uomini e scrittori del mio tempo, p. 207.

24 Le mot arsis est utilisé au sens latin d'ictus, temps fort.

25 Ce type de césure consiste à séparer, dans le dactyle qui forme le troisième pied, les deux premières syllabes (longue puis brève, formant donc un trochée) de la dernière, brève. 


\section{L'importance de l'intonation}

Comme l'a remarqué Pietro Giannini, " pour comprendre pleinement le sens de l'expérience de Pascoli, il faut prendre en considération une autre notion, présente dans la lettre à Chiarini, celle de "récitation convenable" 26 ". Le poète italien affirme que cette démarche avait déjà été adoptée dans l'Antiquité, lorsque coexistaient deux lectures possibles des vers latins, l'une grammaticale et l'autre rythmique. Afin de prononcer rythmiquement le vers, l'oreille ne suffisait pas : il fallait utiliser également le doigt, c'est-à-dire la percussion de l'index. "La "conscience rythmique", comme l'appelle Pascoli, s'est affinée avec le temps, et elle devait être beaucoup plus marquée chez les poètes de l'époque augustéenne que chez les poètes comiques ou chez Ennius ${ }^{27}$." Cette démarche était devenue encore plus importante pendant la latinité tardive, lorsque le sens de la quantité avait cédé la place à l'accent d'intonation : pour marquer l'ictus ou arsis et retrouver donc la mesure du vers, il fallait avoir recours au plausus en frappant des mains, comme on le lit dans le De musica de saint Augustin ${ }^{28}$. Pascoli rappelle que, selon cet auteur, il fallait, pour faire entendre le rythme de la poésie aux oreilles des auditeurs, associer la prononciation d'un homme instruit et expérimenté (docti et exercitati hominis pronuntiatio) et un sens de l'ouïe qui ne fût pas plus lent que ne l'exige la condition humaine (sensus audientium non [...] tardior quam humanitas postulat ${ }^{29}$ ).

Pour restaurer le vers quantitatif ancien dans la langue accentuelle italienne Giovanni Pascoli adopte la solution suivante :

La langue italienne n'oppose pas des syllabes longues à des syllabes brèves, mais elle possède des syllabes qui peuvent être allongées ou non. Et une syllabe peut être allongée avec une intonation convenable, de manière à lui faire atteindre une longueur double de celle d'une brève. Ce faisant, nous sommes dans les mêmes conditions que les Grecs et les Latins ${ }^{30}$.

En s'appuyant sur les travaux de Pietro Rasi ${ }^{31}$, Pascoli attribue à l'arsis une élévation de ton, indiquant par là que la syllabe longue représente un accent mélodique. Ainsi, par une élévation du ton de la voix, les bons lecteurs musiciens peuvent restituer le rythme du vers ancien, de même que dans l'Antiquité, seuls les bons lecteurs musiciens pouvaient être de bons interprètes des vers anciens. Puisque, conclut Pascoli, le texte d'Homère est né du savant mélange de la voix et du vers écrit, parole et chant

26 Giannini, p. 384.

27 Garboli, "Premessa " à Regole..., in G. Pascoli. Poesie e prose scelte, t. II, p. 190.

28 Giannini, p. 384. Cf. Pascoli, Regole..., in G. Pascoli. Poesie e prose scelte, t. II, p. 204-205.

29 Augustin, De Musica, IV, 37.

30 Pascoli, Regole..., in G. Pascoli. Poesie e prose scelte, t. II, p. 273-274.

31 P. Rasi, Dell'omeoteleuto latino, Padoue, 1891. 
doivent s'accorder, et la lecture à haute voix doit faire entendre, aujourd'hui comme dans l'Antiquité, ce que le vers écrit ne peut donner à entendre.

Cela dit, Marco Bianchi a montré que les expériences de traduction de Giovanni Pascoli ne correspondent pas à une rigoureuse application de la théorie présentée dans ses Regole di metrica neoclassica et que, en fin de compte, il vaut mieux lire ses vers en adoptant l'accent italien des mots plutôt que de marquer les mesures de l'hexamètre ancien, comme le poète le recommandait dans la Lettera a Giuseppe Chiarini, car cela empêcherait le lecteur d'apprécier à sa juste valeur la traduction lexicale et syntaxique du vers homérique ${ }^{32}$.

\section{Caractéristiques stylistiques et linguistiques des traductions de l'Iliade par Pascoli}

Le petit volume inachevé des Regole di metrica neoclassica aurait dû comporter, dans un appendice, les Saggi metrici ("Essais métriques »), c'est-à-dire, selon les mots qu'emploie Pascoli lui-même dans une note destinée à les accompagner, " des ébauches de traduction du grec et du latin, [rédigées] non pas comme des modèles à imiter, mais comme des tentatives à améliorer ${ }^{33}$ ". Si ce projet de publication n'a jamais vu le jour dans la forme annoncée, Giovanni Pascoli a publié ses traductions homériques dans l'anthologie scolaire Sul limitare (1900). Ces traductions ont été ensuite rééditées dans le volume posthume Traduzioni e riduzioni sans les introductions et les notes qui accompagnaient leur première parution, mais augmentées d'autres traductions.

L'analyse d'un ensemble cohérent d'extraits homériques traduits par Giovanni Pascoli, provenant du premier chant de l'Iliade ${ }^{34}$, permet de mettre en évidence les caractéristiques stylistiques et linguistiques des traductions en hexamètres de ce poète et notamment la tonalité affective-dramatique, la recherche phonétique et la précision lexicale ${ }^{35}$.

32 Bianchi, p. 14-18 et notamment p. 15.

33 Appendice di note alle "Regole di metrica neoclassica ", in Prose di Giovanni Pascoli, con una premessa di A. Vicinelli, 2 vol., Milan, Mondadori, 1952, t. I, Pensieri di varia umanità, p. 1008.

34 Il s'agit de dix extraits, pour un total de 126 vers : Invocazione alla Musa, $1-7$; Preghiera, 37-42 ; L'arrivo del dio punitore, 44-47 ; L'apparizione, 188-200 ; I due araldi, 327-344; La madre, 345-363 ; Il racconto di Achille, 364-395 et 407-412 ; La risposta della madre, 413-422 ; Invocazione, 451-456 ; Achille adirato, 488-492. Puisque les quatre premiers extraits ne figuraient pas dans l'anthologie Sul limitare, ces textes seront cités d'après le volume posthume Traduzioni e riduzioni raccolte e riordinate da Maria, Bologne, Zanichelli, 1913-1958, p. 3-8.

35 Cf. Devoto, p. 58-59 et Bianchi, p. 19-30. 


\section{La tonalité affective-dramatique}

Un éminent critique contemporain de Giovanni Pascoli, Benedetto Croce, avait affirmé, du vivant du poète, que ce dernier avait «infantilisé " l'esprit homérique ${ }^{36}$. Ce jugement sévère s'explique à la lumière de la poétique du fanciullino, du petit enfant capable de s'émerveiller ; cette poétique, qui fonde et inspire toute la production du poète italien ${ }^{37}$, avait déjà fait écrire à Manara Valgimigli, au sujet des traductions pascoliennes de l'Énéide, que si

[...] Virgile chante guerres et batailles, [chez Pascoli] tout le sens de la merveilleuse épopée est dans le chant matinal d'hirondelles et moineaux qui éveille Évandre dans sa cabane, qui est située là où devaient s'élever les palais impériaux de Rome ${ }^{38}$.

Dans l'épisode évoqué, comme dans les extraits homériques, la valeur donnée au décor naturel des événements confere aux vers de Giovanni Pascoli un ton à la fois affectif et dramatique. La valeur affective des traductions pascoliennes apparait de manière évidente dans l'utilisation des formes nominales dérivées, comme dans le

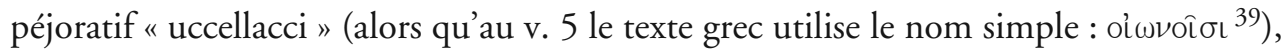
qui permet au poète de mettre en avant la cruauté des oiseaux dévorateurs de héros.

$\mathrm{La}$ "polarisation dramatique-pathétique » des traductions homériques de Pascoli a été soulignée par Marco Bianchi ${ }^{40}$ : d'après ce critique, le souci du poète a été moins de raconter l'Iliade que de présenter à ses lecteurs le personnage d'Achille, avec ses qualités héroïques et son sort tragique. Les extraits de l'Iliade traduits par Pascoli représentent ainsi autant de fragments qui, lus les uns après les autres, composent le portrait du héros, dont le trait essentiel était déjà mis en avant par le titre de la première section de l'anthologie Sul limitare consacrée à l'Iliade: L'eroe del dolore ("Le héros de la douleur $»)^{41}$.

La valeur dramatique de ces traductions apparaît par exemple dans les répliques $\mathrm{du}$ dialogue entre le protagoniste et sa mère (La madre, 345-363 ; Il racconto di

36 Je traduis par ce terme le sens de la critique de Benedetto Croce : «Omero rimbambinito ", citée par Garboli, G. Pascoli. Poesie e prose scelte, t. II, p. 125.

37 Voir Il Fanciullino, a cura e con un saggio di G. Agamben, Milan, Feltrinelli, 1982 (Traduction française : Le petit enfant, par B. Levergeors, Paris, éd. M. de Maule, 2004).

38 Ainsi s'exprime Manara Valgimigli dans Uomini e scrittori del mio tempo, cité par GarBoLI, G. Pascoli. Poesie e prose scelte, t. II, p. 128.

39 Toutes les citations de l'Iliade sont tirées de l'édition de P. Mazon, Paris, Les Belles Lettres, 1996 (1937). Sauf indication contraire, les vers cités proviennent du premier chant de l'Iliade.

40 Bianchi, p. 13.

41 Par opposition, la section de l'anthologie consacrée à l'Odyssée s'intitule L'eroe dell'odio ("Le héros de la haine »). 
Achille, 364-395 et 407-412 ; La risposta della madre, 413-422), qui prennent le ton de répliques théâtrales, grâce notamment à l'utilisation d'exclamations comme les nombreux «oh!» $(414,415,418)$ qui, moins fréquents en grec, ponctuent le texte en italien et soulignent l'emphase introspective, contemplative et pathétique à la fois qui caractérise l'approche homérique de Pascoli. D'ailleurs, afin de faire du lecteur un spectateur de la situation décrite, Pascoli n'hésite pas à ajouter en italien des termes qui permettent de mieux visualiser l'action. Un exemple en est l'adverbe « ecco » (« voici »), sans équivalent dans le texte grec, pour introduire le personnage de Pallas lors de son entrée en scène (194: " ecco, Pallade venne ").

Enfin, le pathos de la situation décrite peut être souligné par la répétition de quelques mots clés, comme par exemple lorsque, en réponse à une demande d'explication de sa mère, Achille répond : "Ma se lo sai, a che mai ragionarti di cio', se lo sai ?"

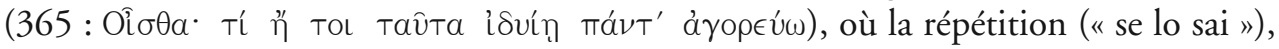
insiste plus que ne le fait le texte grec, par la phonétique aussi (l'allitération en " $\mathrm{s}$ »), sur l'idée de la répétition d'une vérité connue. De même, la répétition des pronoms personnels, comme au v. 393 : "Dunque se pure tu puoi, tu proteggi il tuo nobile figlio ", alors qu'ils peuvent être omis en italien (en grec le pronom est présent mais il

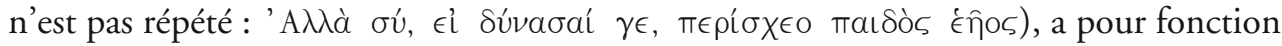
de renforcer le lien entre un personnage et l'action qui lui est attribuée.

Cette tonalité affective-dramatique est due au sens pascolien de l'acceptation de l'Histoire, ce qui ferait penser, si la référence n'était pas anachronique, à une conception girardienne de la vie ${ }^{42}$, selon laquelle le héros accomplit sa destinée en se mettant au service de l'Histoire. Dans la " Note aux élèves " qui accompagnait ses traductions dans l'anthologie scolaire Sul limitare le poète exhortait d'ailleurs en ces termes son jeune lecteur : «[...] sois Achille, mon garçon, sois Achille lorsqu'il s'agit de ton devoir. [...] Rappelle-toi que l'héroïsme ultime ne consiste pas à s'opposer, mais à se résigner (Achille se résigne à la mort), à souffrir plus encore qu'à agir ${ }^{43}$."

Si le travail stylistique de Giovanni Pascoli souligne avec insistance la souffrance du héros, son adaptation de l'Iliade se fonde également sur une recherche d'effets phonétiques, visant à reproduire les échos du drame épique par la musicalité de la langue italienne.

42 Cf. les réflexions de René Girard sur le sacrifice et le bouc émissaire.

43 Pascoli, Nota per gli alunni, in Sul limitare..., p. XIII-XXVIII, notamment p. XVIII. 


\section{La recherche phonétique}

Moins soucieux d'expliquer le vers homérique que d'en restituer la musicalité et le style en italien, conformément à sa conception de la traduction ${ }^{44}$, Giovanni Pascoli utilise, dans ses adaptations homériques, un langage à la fois archaïque et familier.

Un exemple de terme archaïque se trouve au v. 342 : "cotestui ». Mais à y regarder de plus près, les termes qui sonnent comme des archaïsmes sont plutôt des néologismes ou des mots rares utilisés en fonction des exigences métriques, comme « saettame » (382), nom collectif composé à partir du substantif « saette » (385). De même, l'impression de langage désuet vient de l'usage des anastrophes auxquelles le poète est également contraint par la construction de l'hexamètre, comme par exemple

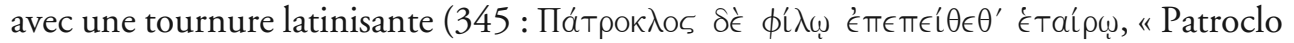
al caro compagno ubbidiva ") et une tournure proleptique comme " mentre gli eroi

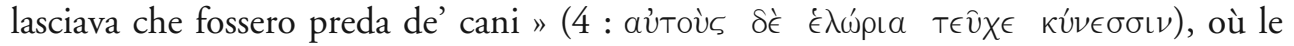
sujet de la proposition subordonnée est inclus dans la principale.

Le langage familier est dû à l'utilisation de formes nominales dérivées, tandis qu'Homère utilise le nom simple, comme dans le cas, déjà cité, du mot "uccellacci " (5), où la charge émotionnelle du péjoratif est accrue par l'allitération en " c " ${ }^{45}$ et par la répétition, originale par rapport au texte grec, d'un adverbe par asyndète, comme au v. 368 : «ben bene " et au v. 391 : « or ora ".

Ces effets de langue ne sont pas rares dans la poésie de Pascoli et notamment dans ses adaptations homériques, où les effets phonétiques reposent sur un système complexe de répétitions. Les figures de rhétorique le plus souvent employées sont l'allitération, l'anaphore et l'anadiplose. Quelques-unes des nombreuses allitérations qui ponctuent les hexamètres pascoliens se trouvent au v. 39 " ch'hai le saette che liberano!» (cheville

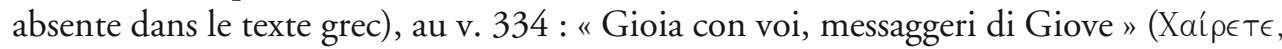

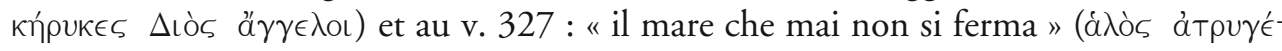
Toto), heureuse formule où la répétition de la syllabe " ma » donne à entendre le flux et le reflux de la vague marine. En dehors des traductions fidèles au texte grec (par ex.

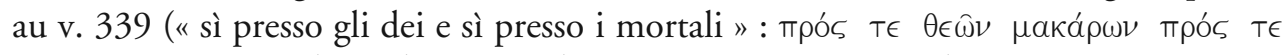
$\theta \nu \eta \tau \omega \hat{~} \nu$ à $\nu \rho \omega \dot{\pi}(\omega \nu)$, l'anaphore pascolienne apparaitt par exemple aux v. 414-415, avec la répétition de l'exclamation " $\mathrm{Oh} !$ " signalée plus haut et au v. 40, " mai, se mai arsi

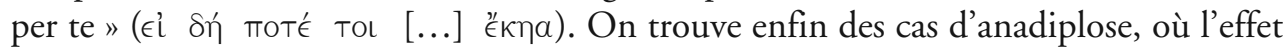
de répétition est ajouté par Pascoli, au v. 329 : «Alla capanna vicino, vicino alla nave

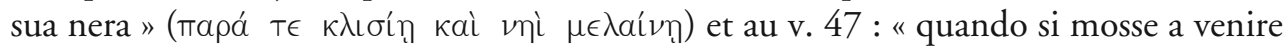

44 Pascoli, La mia scuola di grammatica, in G. Pascoli. Poesie e prose scelte, t. II, p. 13861387.

45 Cet emploi de la langue a éveillé la sensibilité de Pier Paolo Pasolini : lecteur attentif de Pascoli, il lui avait consacré son mémoire de maîtrise, et il lui a très probablement emprunté le péjoratif " uccellacci » pour lui opposer, dans le titre d'un film célèbre, le diminutif « uccellini ». 


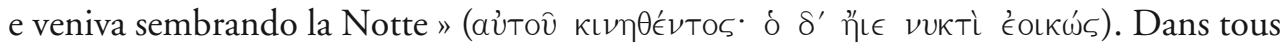
ces cas, la répétition d'un substantif, d'un verbe ou d'un adverbe ne vise pas seulement à souligner l'idée exprimée : elle confere un effet musical et un caractère solennel à la prosodie épique.

Au début du $\mathrm{Xx}^{\mathrm{e}}$ siècle ces préciosités linguistiques étaient dans l'air du temps et elles n'avaient pas manqué de susciter l'intérêt des poètes de l'époque, en particulier de Gabriele D'Annunzio, qui, dès 1896, avait d'ailleurs sollicité l'aide de Pascoli pour une traduction rythmique du chœur de l'Antigone de Sophocle, et à qui Pascoli avait pensé, dans un premier temps, dédier son manuel de métrique ${ }^{46}$.

Bien que les adaptations homériques de Pascoli aient une indéniable qualité affective-dramatique et musicale, c'est dans le rendu lexical de la langue grecque en italien que la prosodie des hexamètres italiens atteint ses résultats les plus remarquables.

\section{La précision lexicale}

Quelques exemples tirés du corpus des traductions choisies peuvent illustrer le traitement sémantique de la langue d'Homère par Giovanni Pascoli. Ses choix lexicaux témoignent en particulier d'un souci étymologique et d'un approfondissement conceptuel ${ }^{47}$.

La qualité la plus importante des expériences homériques de Pascoli consiste dans la rigueur philologique de sa traduction. La précision littéraire apparaît par exemple dans la traduction des patronymes et des épithètes homériques. Le fameux patronyme d'Achille, au premier vers de l'Iliade, est traduit fidèlement par le terme "Peleìade ", de même que le patronyme d'Agamemnon trouve son correspondant dans le patronyme «l'Atride » (191). On remarque un traitement analogue, très fidèle à l'original grec, des

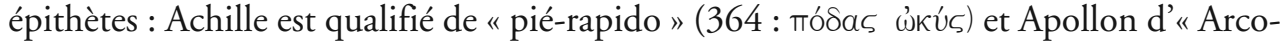
d'argento " (37 : 'Арүvро́то $\left.\xi^{\prime}\right)$. Le trait d'union, souvent utilisé alors par Pascoli, a la fonction de rappeler la présence d'un mot composé.

La rigueur philologique pousse Pascoli à chercher, dans la racine du mot grec, le sens originel de ce dernier, de manière à choisir le terme le plus adapté pour le traduire

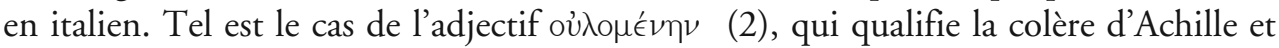
qui contient l'idée de la destruction, si importante dans le prélude du poème épique. Le terme " funebre " proposé par Pascoli rend en effet le sens du terme grec correspondant : ö $\lambda \lambda \vartheta \mu \iota$ ("faire périr, détruire, anéantir »). De manière analogue, Pascoli traduit le verbe grec $\phi \theta \iota v \dot{\theta} \theta \in \sigma \kappa \in$ (491) par « macerava », afin de rendre sensible, en italien aussi, l'effet de la rage sur le cœur d'Achille.

46 V. Garboli, "Premessa " à Regole..., in G. Pascoli. Poesie e prose scelte, t. II, p. 180-183.

47 L'analyse sémantique des traductions de l'Iliade par Giovanni Pascoli a été conduite de manière plus détaillée, sur un autre ensemble de péricopes, relatives au livre 22, par BIANCHI, p. 18-30. 
Pascoli témoigne également d'un souci d'approfondissement conceptuel dans la construction de certains qualificatifs dont il souhaite renforcer l'idée. Dans la traduc-

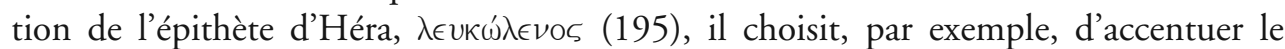
blanc, pour faire ressortir l'idée de lumière que le mot $\lambda \in$ UKós comporte : «la Braccia-

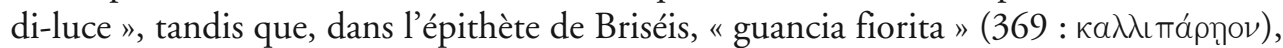
c'est la qualité de la beauté contenue dans le terme d'origine qui est justifiée et même expliquée par l'image de la fleur épanouie.

À la fin de cette rapide présentation des principales qualités stylistiques des traductions de Pascoli, deux remarques s'imposent : d'un côté, le traitement hyperbolique des émotions par le poète italien permet de comprendre la critique que lui avait adressée Benedetto Croce d'avoir "infantilisé " l'esprit homérique ; inversement, le travail rationnel du poète, qui prend racine dans l'étude philologique de la langue et dans l'approfondissement conceptuel, ne peut que faire reconnaitre dans ces traductions, comme l'a fait Marco Bianchi, un Homère plus que jamais « adulte ${ }^{48}$ ".

\section{Nouvelles voies et voix nouvelles de l'hexamètre dactylique en italien}

D'après les affirmations du poète lui-même, les expériences de traduction en hexamètres de Giovanni Pascoli étaient destinées à évoluer ${ }^{49}$. Mais plutôt que d'améliorer ses vers, comme il l'avait annoncé, Pascoli finit par abandonner non seulement ce projet, mais aussi celui de publier les versions intégrales des Regole di metrica neoclassica et de la Lettera a Giuseppe Chiarini. Chez ce poète, l'hexamètre italien connaitra une nouvelle vie dans des traductions d'autres auteurs, avant d'être définitivement délaissé en faveur de l'hendécasyllabe libre. Mais l'hexamètre en italien n'a pas pour autant épuisé ses ressources, puisque deux nouvelles traductions de l'Iliade, l'une de Manlio Faggella $^{50}$ et l'autre de Daniele Ventre ${ }^{51}$, rédigées toutes deux en hexamètres dactyliques, ont vu le jour à partir des tentatives pionnières de Giovanni Pascoli. L'histoire de l'hexamètre italien n'est donc pas finie.

\section{Retractatio}

Dans une lettre envoyée le 7 octobre 1899 au dédicataire de ses règles métriques de goût classique, Pascoli avait reconnu que ses traductions ne le satisfaisaient pas

48 BianCHI, p. 11-12.

49 Pascoli, Regole..., in G. Pascoli. Poesie e prose scelte, t. II, p. 179.

50 Omero. Iliade, traduzione in versi esametri di M. Faggella, 2 vol., Bari, Laterza, 1923.

51 Omero. Iliade, traduzione di D. Ventre, Palerme, Mesogea, 2010. 
complètement, et même que les vers étaient presque tous faux ${ }^{52}$. L'analyse de l'évolution de l'hexamètre entre les premiers et les derniers chapitres homériques de l'anthologie Sul limitare devrait permettre de vérifier cette affirmation ${ }^{53}$. D'ailleurs, si l'on considère que, pour Pascoli, la beauté de l'hexamètre est due à une "proportion variée de dactyles et de spondées ${ }^{54}$ ", il est légitime de penser que " la modification essentielle des traductions homériques aurait concerné l'équilibre entre dactyles et spondées 55 \%. Mais le problème que Pascoli repère dans son travail d'adaptation est plus complexe, et il trouve confirmation dans une déclaration que le poète avait faite à ses élèves de grammaire lors de sa dernière année d'enseignement (1910-1911), lorsqu'il leur avoua qu'il ne considérait plus comme valable le système qu'il avait adopté dans ses traductions du grec en hexamètres ${ }^{56}$.

Ainsi, plutôt que d'améliorer ses premières traductions du grec homérique, Pascoli décidait d'abandonner le projet de publier dans sa version intégrale le volume annoncé à Chiarini ${ }^{57}$, et mettait en place un nouveau système d'adaptation de l'hexamètre, qu'il appliquait à deux compositions latines qu'il avait lui-même rédigées en hexamètres, Hymnus in Romam et Hymnus in Taurinos ${ }^{58}$. À la fin de sa carrière d'enseignant, en 1911, convaincu que le vers le plus propre à traduire l'hexamètre en italien était l'hendécasyllabe, et que trois hendécasyllabes représentaient une succession de douze temps forts, avec césure au milieu du deuxième, Pascoli a établi qu'ils représentaient approximativement le rythme et le contenu de deux hexamètres. Il décida donc de traduire ses poèmes de manière à remplacer deux hexamètres par trois hendécasyllabes, avec un nombre total d'ictus inchangé ${ }^{59}$. Voici, par exemple, les deux premiers hexamètres de l'Hymnus in Romam et leur traduction en hendécasyllabes libres :

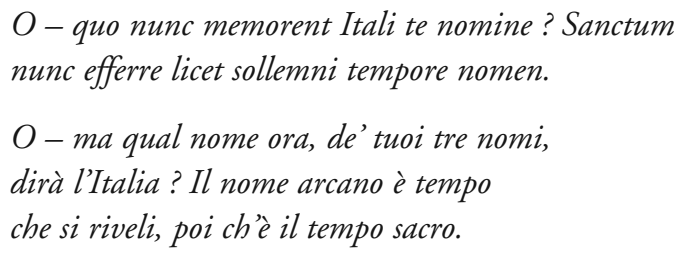

Mais si, pour Pascoli, la traduction de quelques centaines de vers représentait un exercice métrique intéressant, faire une nouvelle adaptation des extraits homériques

52 Pascoli, Regole..., in G. Pascoli. Poesie e prose scelte, t. II, p. 179.

53 Cette suggestion a été proposée par BianCHI, p. 16.

54 Regole..., 54.

55 BIANCHI, p. 17.

56 Giannini, p. 395.

57 Voir A. Traina, " "Retractatio” pascoliana ", Rivista pascoliana 15 (2003), p. 179-182.

58 Pour le texte intégral de ces poèmes, voir G. Pascoli. Poesie latine, a cura di M. Valgimigli, Milan, Mondadori, 1951-1961.

59 Audisio, p. 68-70 et Bianchi, p. 18. 
traduits en hexamètres a dû lui paraittre un projet trop ambitieux pour être entrepris ${ }^{60}$. Pascoli se lança donc dans un nouveau recueil, les Conviviali ; ce sont des poèmes consacrés entre autres à des personnages homériques; l'hendécasyllabe libre y occupe désormais la place que l'hexamètre avait tenue dans les traductions des poèmes grécolatins et dans ses œuvres latines ${ }^{61}$.

\section{La nouvelle vie de l'hexamètre chez Pascoli et chez les autres traducteurs de l' Iliade}

Giovanni Pascoli utilise l'hexamètre non seulement pour traduire des extraits d'Homère et de Virgile ${ }^{62}$, mais aussi pour rédiger toute une série de poèmes latins ${ }^{63}$, très appréciés à son époque ${ }^{64}$. De plus, il emploie ce même vers dans la traduction d'un texte anglais moderne d'inspiration homérique, celui qu'Alfred Tennyson consacre, en pentamètres iambiques, au retour d'Ulysse chez les siens ${ }^{65}$. Ce poème (Ulisse) a été traduit et inséré par Pascoli dans son anthologie Sul limitare (p. 399-400), avant de paraître dans le recueil posthume Traduzioni e riduzioni ${ }^{66}$.

Il est tentant de voir, dans les derniers vers du poème, cités en exergue de cette étude, une image de la mission du poète à laquelle Giovanni Pascoli aurait pu adhérer, dans un élan qui l'aurait porté à s'identifier, voire à se confondre, avec le personnage homérique. Le vers final ("sempre in lottare e cercare e trovare né cedere mai ») semble d'ailleurs reprendre et compléter le contenu d'une note qui apparaît à la fin de l'anthologie Sul limitare, où le poète souhaite " achever, c'est-à-dire recommencer ${ }^{67}$ " son œuvre, à la manière du chant éphémère d'un aède.

Bien que complexe, problématique et frustrant, le travail de Giovanni Pascoli a permis de faire avancer la réflexion sur la traduction des poèmes homériques en hexamètres italiens, en particulier de l'Iliade. Deux autres versions ont été proposées depuis

60 J'emprunte les lignes directrices de cette réflexion à Marco Bianchi, ibidem.

61 Cf. E. Bigi, "La metrica delle poesie italiane di Pascoli », Giornale Storico della Letteratura Italiana 412 (1958) p. 552-586.

62 L'ensemble de ces extraits a été publié par le poète dans l'anthologie scolaire Sul limitare... (p. 452-491), tandis qu'un choix restreint en a été réédité dans le volume posthume Traduzioni e riduzioni..., p. 132-138.

63 Il s'agit des recueils suivants : Liber de poetis (1891-1910), Res romanae (1892-1906), Poemata christiana (1901-1911), Hymni (1911) et Ruralia (1893-1899).

64 Ces poèmes ont obtenu de nombreux prix internationaux de poésie. Les cent premiers vers de l'Hymnus in Romam, sous le titre de Carmen, ont été présentés au concours pour le premier cinquantenaire de la République italienne. Pascoli remporta le deuxième prix (le premier ne fut pas attribué).

65 A. Tennyson, Poems, 1842.

66 Traduzioni e riduzioni..., p. 179-180.

67 Sul limitare..., p. 608. 
la mort du poète. Au lendemain de la Première Guerre mondiale, Manlio Faggella a réalisé le projet d'imiter les traductions allemandes et anglaises des poèmes homériques en hexamètres (il cite dans l'introduction ses modèles, Voss et Longfellow). Dans sa traduction de l'Iliade ${ }^{68}$, Faggella, peu connu de la critique ${ }^{69}$, se démarque surtout de l'exemple métrique de Vincenzo Monti ${ }^{70}$. Il était soucieux de donner à l'Italie sa première version de l'Iliade en hexamètres, alors que presque tous les pays européens, Slovénie et Finlande comprises, possédaient déjà la leur. Sa prosodie est marquée par une recherche lexicale philologique (par ex., l'épithète d'Héra, 195, est rendue par «dalle candide braccia »), par un lexique parfois archaïsant (5: « convito »; 39 : " delubro »), parfois éloigné de sa source (5: "corvi ») et par de claires réminiscences pascoliennes (307: " guancia fiorita "; 334 : "Gioia con voi, messaggeri »).

Presque cent ans après cette première expérience de traduction intégrale de l'Iliade en hexamètres italiens, Daniele Ventre en a proposé une nouvelle ${ }^{71}$. Les principales qualités de cette adaptation sont sa fidélité littérale au texte d'origine (ex. : 2 : « devastatrice "; 5 : " uccelli "; 195 : "bianca di braccia »), son recours au lexique italien contemporain ( $4:$ : razzia »; $5:$ «banchetto »; $7:$ "splendido Achille ») et sa syntaxe fluide, qui évite, autant que possible, les constructions anastrophiques (4: «[...] delle spoglie imbandì razzia per i cani ") et fait de la prosodie homérique un récit presque oral, ce qui le rapproche de ses origines.

Les essais de traduction isométrique des poèmes d'Homère en italien par Giovanni Pascoli offrent un témoignage précieux de la volonté de ce poète de restaurer, dans la langue accentuelle italienne, l'ancienne quantité rythmique de l'hexamètre antique, de manière à déformer le moins possible sa structure métrique d'origine, afin qu'il n'apparaisse pas ridicule ou gauche aux oreilles d'auditeurs modernes ${ }^{72}$.

Telle a été en effet l'ambition de Giovanni Pascoli : recréer, plutôt qu'interpréter. Alors que la traduction ut interpres permet d'expliquer ce que veut dire le vers étranger, la traduction ut orator vise, d'après l'" école de grammaire » de Giovanni Pascoli, à faire

68 Manlio Faggella est également l'auteur d'une traduction intégrale de l'Odyssée en hexamètres italiens (1925).

69 Un colloque et un livre lui ont été cependant consacrés : Manlio Faggella e gli studi classici italiani, Atti del Convegno nazionale di studi lucani, San Fele, 29 settembre 1983, a cura di F. Noviello, Venosa, Osanna, Collana di studi lucani e meridionali, 1985.

70 Faggella, « Prefazione », p. VII. Vincenzo Monti avait traduit l'Iliade en adoptant l'hendécasyllabe libre (1811-25).

71 Ce travail a été couronné par un prix de traduction (Premio "Achille Marazza ») en 2011.

72 Voir Pascoli, La mia scuola di grammatica, in G. Pascoli. Poesie e prose scelte, t. II, p. 1385. 
entendre, par une traduction fidèle, non seulement les aspects linguistiques et stylistiques du texte grec, mais également ses aspects rythmiques et métriques ${ }^{73}$.

L'originalité des expériences pascoliennes apparaît à la lumière des traductions en hexamètres italiens de l'Iliade qui les ont suivies. La version de Manlio Faggella et, plus encore, celle de Daniele Ventre, doivent beaucoup aux innovations courageuses, mais plus ou moins satisfaisantes, tentées par Giosué Carducci et Giovanni Pascoli.

Il serait alors souhaitable, comme l'a déclaré Pietro Giannini ${ }^{74}$, de mettre les traductions métriques pionnières de Giovanni Pascoli à leur juste place, au sein des réflexions sur les possibilités rythmiques des langues accentuelles, et de leur donner enfin le statut d'œuvre poétique qu'elles méritent, par une organisation rationnelle et une édition bilingue des extraits traduits.

Mais s'il est vrai, comme le répétait sans cesse Giovanni Pascoli, que le poète est un aède et donc un orateur, les tentatives de restitution du vers homérique par Giovanni Pascoli, Manlio Faggella et Daniele Ventre trouveraient leur véritable aboutissement dans un travail de mise en scène dramatique analogue à celui que Philippe Brunet applique à sa traduction de l' Iliade $^{75}$ en hexamètres français. Là, à travers la performance orale et l'action dramatique, la puissance de la parole s'accorde finalement à la cadence du pied et le vers homérique retrouve les composantes de son charme.

\begin{tabular}{l} 
Cristina NOACCO \\
\hline PLH-ERASME (EA 4601) \\
Université de Toulouse-Jean Jaurès \\
5, allées Antonio Machado \\
F.31058 Toulouse cedex 9 \\
cnoacco@yahoo.fr
\end{tabular}

\section{Bibliographie sélective}

F. Audisio, "Metrica "neoclassica" e metrica italiana ", La rassegna della letteratura italiana 3 (settembre-dicembre 1995), p. 34-91.

P. G. Beltrami, La metrica italiana, Bologne, Il Mulino, 1994 (1991).

M. BiAnCHI, "Oltre il limitare : l'Omero "adulto" di Giovanni Pascoli. Le traduzioni pascoliane dell' Iliade e la questione della metrica neoclassica ", Rivista pascoliana 23 (2011), p. 9-30.

E. BIGI, «La metrica delle poesie italiane di Pascoli », Giornale Storico della Letteratura Italiana 412 (1958), p. 552-586.

73 Ibid., p. 1386-1387.

74 Giannini, p. 395-396.

75 L'Iliade. Homère, traduit du grec par Ph. BRUnet, Paris, Seuil, 2010. 
G. CARDucCI, Odi barbare, introduction, notices et commentaires par A. Ricklin, Paris, Les Belles Lettres, 1950.

G. ConTINI, «Innovazioni metriche italiane fra Otto e Novecento ", in ID., Varianti $e$ altra linguistica. Una raccolta di saggi (1938-1968), Turin, Einaudi, 1970, p. 587-599.

B. Croce, G. Pascoli, Bari, Laterza, 1956 (1920).

G. Devoto, «Problemi delle traduzioni pascoliane», Studi per il centenario della nascita di G. Pascoli pubblicati nel cinquantenario della morte. Convegno bolognese (28-30 marzo 1958), 3 vol., Bologne, Commissione per i testi della lingua, 1962, t. II, p. 57-67.

M. FAGGella, Omero. Iliade, traduzione in versi esametri, 2 vol., Bari, Laterza, 1923.

N. Gardini, "An Ancient Soul in a New Body : Giovanni Pascoli's Homeric Translations ", Italian Studies 66/1 (2011), p. 59-75.

M. Geymonat, "Osservazioni sui primi tentativi di metrica quantitativa italiana ", Giornale Storico della Letteratura Italiana 143 (1966), p. 378-389.

P. Giannini, "Le traduzioni "metriche" di G. Pascoli ", in Teorie e forme del tradurre in versi nell'Ottocento fino a Carducci, Atti del convegno internazionale, Lecce, 2-4 ottobre, a cura di A. Carrozzini, Galatina, Congedo Editore, 2010, p. 379-396.

G. Pascoli, Sul limitare. Prose e poesie scelte per la scuola italiana, Milano-PalermoNapoli, Remo Sandron Editore, s. d., $4^{\mathrm{e}}$ éd. (1 $1^{\mathrm{re}}$ éd. : 1900).

G. Pascoli, Poesie e prose scelte, éd. C. Garboli, Milan, Mondadori, 2002.

E. PASQUINI, "Pascoli vs Carducci : due modalità di traduzione ", in Teorie e forme del tradurre in versi..., p. 363-377.

P. Rasi, Dell'omeoteleuto latino, Padoue, 1891.

A. TraInA, "Retractatio" pascoliana ", Rivista pascoliana 15 (2003), p. 179-182.

M. Valgimigli, " Nascita dell'esametro pascoliano ", in ID., Uomini e scrittori del mio tempo, Florence, Sansoni, 1965, p. 211-214.

D. Ventre, Omero. Iliade (traduzione), Palerme, Mesogea, 2010. 